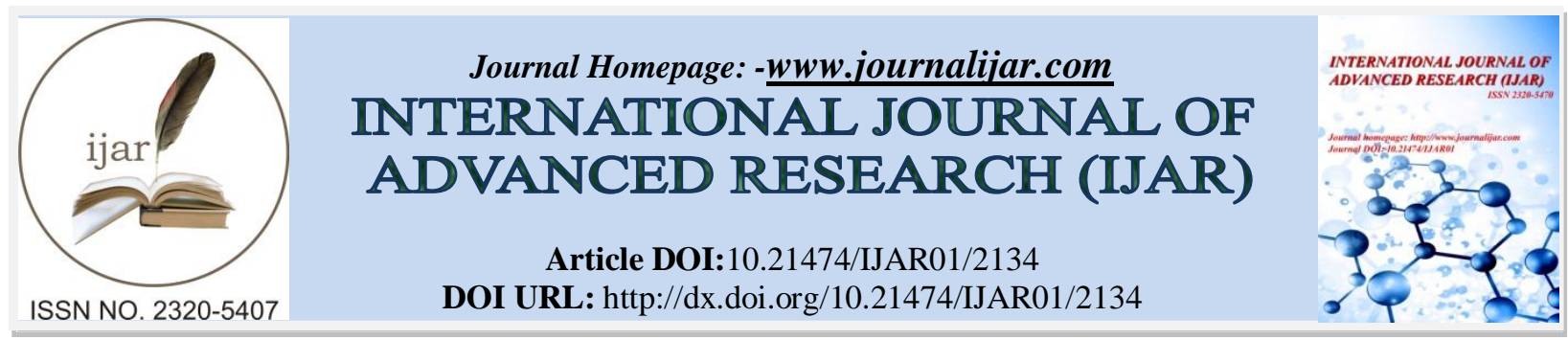

RESEARCH ARTICLE

\title{
BUSINESS INTELLIGENCE: FROM WHAT IT IS TO HOW IS IT MAKING CITIES SAFER TO LIVE.
}

Raghav Luthra $^{1}$, Meenu Chhabra ${ }^{1}$, Angad Deep Singh Nanda ${ }^{1}$ and Mohit Tiwari ${ }^{2}$.

1. Bharati Vidyapeeth's College of Engineering, New Delhi.

2. Dept. of Comp. Sc, BharatiVidyapeeth's College of Engineering, New Delhi.

\section{Manuscript Info}

Manuscript History

Received: 25 September 2016

Final Accepted: 27 October 2016

Published: November 2016

Key words:-

Business Intelligence, Criminal analysis, Intelligence Analysis, Public safety, Tableau

\section{Abstract}

This paper aims to discuss what Business Intelligence is and how a public safety model is made and implemented to make any city or country safer to live. Using technology to decrease crime rates is now a common practise in some cities. Business Intelligence also participates in the same with its tools and techniques. The paper will analyse a working model of an open system of the city Baltimore, Maryland where we can access and visualise crime records in a very interactive manner. Interactive dashboards of Delhi, the National Capital Region of India, from the crime data which includes number of different crimes occurred in each state and union territories from 2001 to 2012 have been created to give a glimpse of a new public safety model. We will discuss the future scope on how the crime data can be categorized and visualised efficiently for better analysis and hence improve the safety of public.

Copy Right, IJAR, 2016,. All rights reserved.

\section{Introduction:-}

The increase in criminal activities is proving to be a setback for the society. In order to deal with it, it is important to know what kind of offense is done and which area is worst affected. Recent articles on how technology, especially Business Intelligence, has helped decreased crime rate and made the world a safer place $[1,2,6]$ has prompted city governments to make all data public and open for criminal and intelligence analysis.

Firstly, this paper helps in understanding the concept of Business Intelligence along with its importance to public safety is important. This simply gives us a clear and accurate vision of the data which will help in better decision making. Secondly, we discuss about the concept and necessary features required for any public safety model i.e. to create a Public safety model, some datasets like arrest records, emergency call records, trial records, department wise records should be present at each level, ranging from a district to the whole country. These should be open for managing, query and reporting purposes to draw out facts and figures. Many US cities have already taken up the initiative to make an open Public safety model to use and open for discussion. Likewise, a case study of Baltimore city has been taken and discussed. However, it has also been observed that in India, only data is present as a whole and there is no analysis being done for the current condition of the criminal activities done in any Indian city. Hence, interactive graphs for Delhi has been made for the years 2001-2012 using Tableau, one of the best data visualisation software for Business Intelligence.

Corresponding Author:- Raghav Luthra.

Address:- Bharati Vidyapeeth's College of Engineering, New Delhi. 
The paper concludes with a suggestion of few report categories and their respective attributes that might be helpful to increase the data granularity of the department data and make it possible for better analysis. All this will help police narrow down areas of high criminal activity along with the type of crime being taken place

\section{Business Intelligence:-}

Business Intelligence (BI) refers to processing large amount of raw data and converting it into meaningful and useful for business analytics purpose [3]. It helps to store, gather, process and analyse corporate data. A large amount of data is interpreted easily using BI. Strategic business decisions including priority and positioning can be done via business intelligence. Also, it can handle large amount of structured and unstructured data to create better business opportunities. It is useful when both internal and external data about the company is combined and a strategy is made to remove uncertainty in making strategic decisions.

BI combines large set of applications like ad hoc analysis and querying, online analytical processing (OLAP), enterprise reporting, mobile BI, real world BI, location intelligence etc. Today's business has access to more data. Companies produce collect and store large amount of data from customer's feedback to surveys to manufacturing and delivery statistics. Therefore, BI is used to put these methodologies that put this data to work, helping business to become more effective and profitable.

\section{Components of Business Intelligence:-}

Business Intelligence has five main primary components [4] viz.

1. Data Sources: Data sources are the first and foremost component of BI. This includes the capturing of raw data from various sources and collecting them into tables, usually spreadsheets or unstructured data, usually multimedia or text files. Further, BI tools can form various charts like bar graphs, pie charts which can be further used by the organization to develop strategies or goals.

2. Data Warehousing and Data Marts: Data Warehouse is a center where data from various heterogeneous sources is collected and stored. General Extract-Transform-Load (ETL) systems are put before saving data to a warehouse i.e. the data is extracted from a source (homogenous or heterogeneous), transformed according to the need and loaded into the data warehouse. Data Mart, on the other hand, is a unique subset of data warehouse which are made according to a particular area of interest or requirement for the business. Any business organization can now go through their large amount of data from the past using the required subset of data warehouse, which is, a data mart for making better decisions.

3. Online Analytical Processing: OLAP comes in handy when the analysis to be done is multidimensional in nature. General OLAP analysis includes rollup, drill down and slicing and dicing. To 'rollup' means to aggregate the given data into factors necessary while 'drill down' means how the analyst will navigate in and out of the data to draw statistics. The process of slicing and dicing involves 'slicing' the data i.e. creating multi dimensions of the data and then 'dicing' i.e. viewing the data through different views. OLAP makes the data accessible at a faster pace and helps perform powerful calculations

4. Advanced Analytics: Advanced Analytics is the component in which data is analysed, some statistical inferences are made along with prediction models being generated to estimate the future figures. These also include the practise of data mining, text mining, optimization, forecasting, simulation. Hence, this helps business organizations see the direction in which they are moving and predict their state in the future.

5. Real time BI: Real time BI is among the most popular component of all. Now, various business organization can analyse the data in real time via email, messaging etc. Some analytics software's can track live data and create interactive dashboards instantly. These are generally found where time limited activities such as website traffic, surveillance systems etc. need to be monitored all the time.

\section{Importance in Public Safety:-}

- All the decisions are made based upon the real facts: The data of the past experience is collected. These facts are used in better decision making. As the data obtained is analysed first, the decisions made are better than just mere assumptions.

- Accurate reports: The analysis of data provides an accurate report of the crimes happening. These reports can be used to form a pattern and provide better security measures and reducing crimes.

- Protection and Defending by Predicting and Preventing: Once the reports are prepared based on the crime records of an area and a pattern is formed, this pattern can be used to predict the type of crimes that might happen at a given place. Hence, better security can be implemented at that place and people can be protected in a better manner. 
- Proper allocation of resources: All the resources such as money, manpower etc. can be allocated to different places according to the crime rate of that place. This will help in optimizing the resources and help in increasing the safety of the people.

- Using past experience for a better future: The past issues and mistakes are analysed so that such problems can be overcome in the future and better security is provided.

- Data Sharing: All the analysed data, reports and the patterns can be shared to different organizations so that general awareness can be increased and people can protect themselves.

\section{Public safety model:-}

A Public safety model is judged purely by the level of granularity of dataset as well as the features provided by the same on the BI platform. But before that, the concept of each Public Safety model is important to understand that how the police actually use intelligence to draw facts on their own to arrest criminals or utilise manpower.

\section{Concept:-}

The integration of intelligence analysis and crime analysis has helped police departments reduce and prevent further the various criminal activities in the neighbourhood. Also, better law enforcement tactics have been deployed due to this only. This integration is called Criminal Intelligence [7]. Therefore, Intelligence Analysis and Criminal Analysis are the two main concepts behind the Public Safety model.

\section{a. Intelligence Analysis:-}

Gathering intelligence and using intelligence are two different tasks. Intelligence analysis is the process where any information or knowledge product is analysed for gathering new information. This could vary from a call recording to any presentation made. Intelligence analysis is of three types: Tactical, Operational and Strategic.

- Tactical intelligence is used to implement tactical plans which are case specific like chasing an arrest or instantaneously responding to a crime event.

- Operational intelligence is a broader concept and implemented at the organization level. This deals with all kinds of resource utilization and optimal use of man power.

- Strategic intelligence is the type in which analysts understand the patterns and environment of crimes for a period of time and provide their findings. This is seen as a long-term objective to achieve at organizational level.

\section{b. Criminal Analysis:-}

Crime analysis involves the analysis of crimes and other police related issue for evaluation, reduction and prevention of crime [9]. It has three different components same as intelligence analysis i.e. Tactical, Operational and Strategic.

- The tactical aspect focuses on immediate issues that are important to the police.

- The operational issues describe the priority areas and the potential threats.

- The strategic component describes the long-term problems which can be solved by the police department or by non-law enforcement organizations like the city council.

\section{Features:-}

The Public Safety model needs to have a definitive structure right from how the data is stored into the warehouse, loaded via ETL scripts into a BI platform and further used for reporting, managing performance, query and analysis [8].

1. Performance Management: Managing the performance via live dashboards or scorecards is an important aspect. Dashboards provide the real-time scenario to any BI model. In any public safety model, say of a Police Department, dashboard might consist of current number of 1-0-0 calls in progress along with precise location of the origin, the availability of PCR vehicles around, the number of pending calls etc.

2. Scorecards are an alternate version for dashboards. Here, we analyse the whole schedule over a very brief period of time that just passed.

3. Reporting: The model should be created in such a way that it is easy to generate reports out of it to any authorized personal. Reports prepared when there is a need to publicize the facts and findings of the data in various public articles is called embedded report. However, when the report is prepared for the need of any other organization to showcase their results is called enterprise reporting. 
4. Query and Analysis: Queries and analysis is usually done for various ad hoc queries for specific investigation purpose and analysis is done to visualise the whole scenario and help make future decisions.

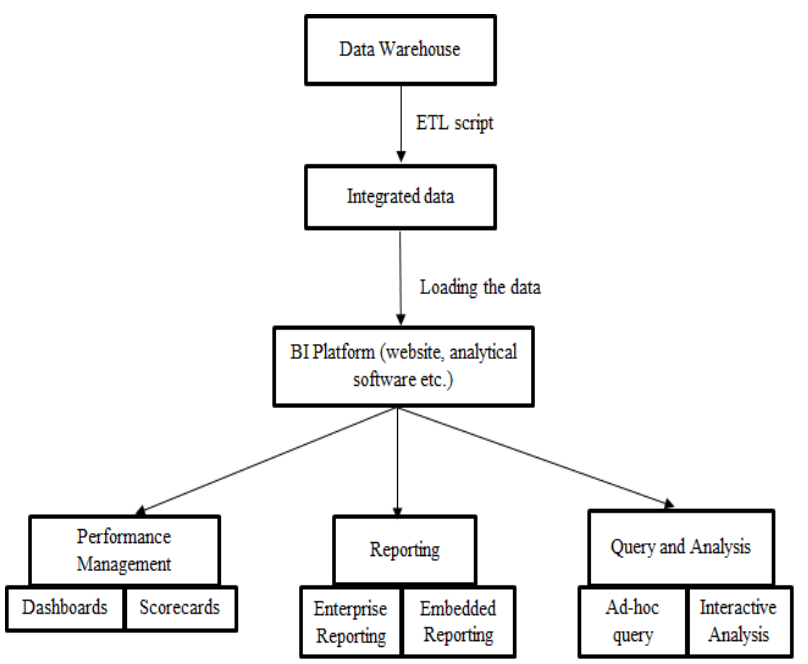

Fig 1:-From loading the data to analysing the data

\section{Baltimore's model:-}

Open data is all about collaboration of developers, people and the jurisdiction. When the Baltimore Police Department Arrest Data [5] website is fully loaded, the dataset is loaded automatically and sorted via Arrest Date. We can see all the attributes like arrest no., sex, age, race, arrest location, incident occurred, charge filed etc.

Also, all 9-1-1 calls dataset is recorded and loaded into the website platform and frequently updated to another Calls for Service dataset [10]. This includes the call time, district, caller number, assigned priority of the call, location of incident etc.

Each record is uniquely defined and can be viewed individually by accessing the "View as rich list" option. Following is the list of functions that the model could perform:

- Any attribute of the dataset could be filtered or sorted according to the need via the Filter tab as well as the menu option of each column/attribute.

- A search bar to find anything in the dataset

- Save the current view of the data after performing required analysis.

- The subset data could be downloaded into many major output formats like csv, json etc.

- Visualising the data on charts and maps.

- Viewing a particular instance of the dataset, generally for a specific motive, by any signed-in user

- Discuss tab to let the users communicate with each other via comments to discuss any view or view related queries

We have seen how transparent and efficient the system is as the police departments can simply create maps and charts to analyse the hotspots of the city and utilize more resources there. Example, a heat map can be created of the city classified according to the age and find where juvenile crimes happen the most. 


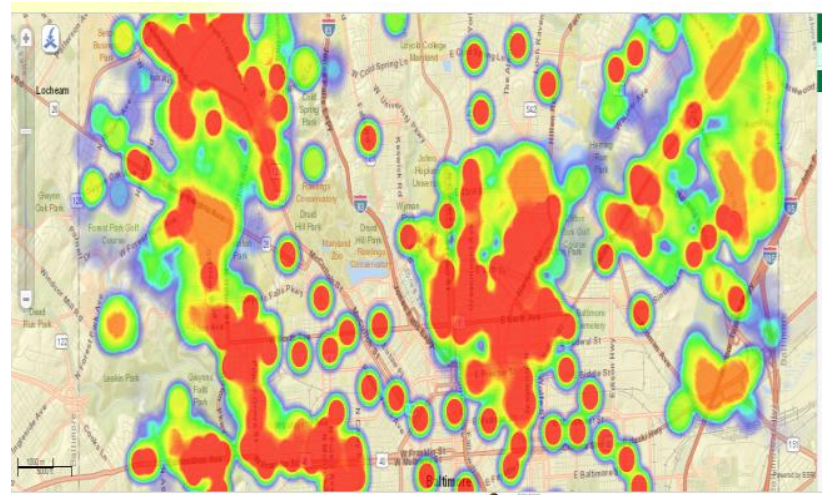

Fig 2:- Heat map of age-wise arrests done in Baltimore

Similarly, mapping the areas where the most number of arrests or 9-1-1 calls have taken place will help police department to utilize their resources in that region will make the system more efficient.

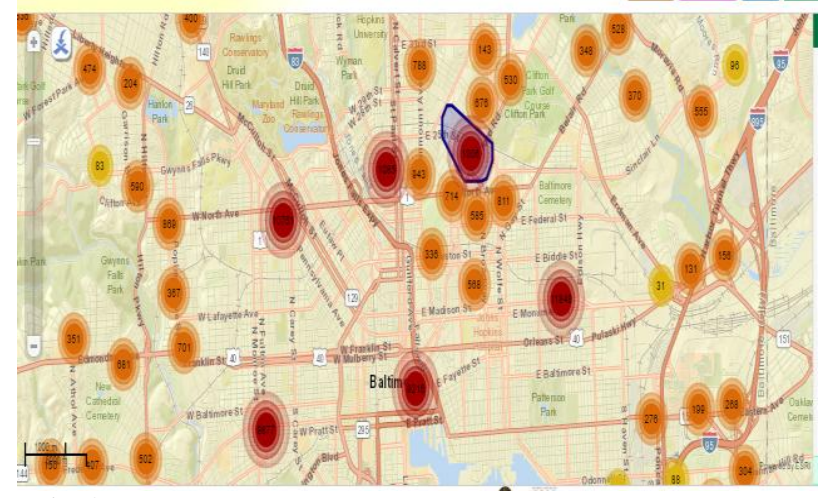

Fig 3:-Number of arrests made per locality in Baltimore

After visualising the data and analysing it, Baltimore's Public Safety model is a total success. It's not only Baltimore which has this model. Various cities like Boston, Chicago, Seattle, Los Angeles have made their dataset public and open for analysis. For the government, this has actual economic benefits like transparency and accountability while the citizens can be directed what to do and what not to do [11]. But, there is always a down side for everything. Open data will pose security issues to the public since personal information can be leaked via these resources.

\section{Proposed work of Delhi using Tableau:-}

Now that we have seen the power of BI when applied on a crime dataset, we believe that its tools have a lot to offer and should be used to make our society safer!

But the data available on Open Government Data (OGD) was just a summary of all the crimes throughout India. The data originally contained the number of Indian Penal Code violations distributed across each state and its district from 2001 to 2012 [15]. The file had attributes like State, District, Year, Number of burglaries, Number of rapes, Number of murders, Total IPC crimes etc.

We made some interactive dashboards of Delhi using Tableau that how it will try to reduce and prevent crime in the nearby future. The same has been uploaded on Public edition of Tableau [12]. Firstly, the crime heads were categorized into 4 general crimes viz.:

1. Crime against women like rape, dowry deaths, assaults, insults etc.

2. All kinds of theft like auto theft, robbery, burglary, dacoit etc.

3. Social crimes like murders, riots, breach of trust, cheating etc.

4. Other crimes like arsons, counterfeiting, death by negligence etc.

Now, by looking at the time series graph for each type of crime in Fig 4, the concerned department can follow the trend line and expect the number of crimes of that category in the future. A good fit of the model will be the one 
with low p-value (indicating high confidence of the null hypothesis) and high R-squared value (indicating high coverage of the variability in the data). R-squared value of 0.766 and p-value of 0.0001 were very good for the crime against women which indicate that if the policies or law remain the same, the crime against them will definitely rise in the nearby future. Some failures were also found like in the case of social crime where p-value was as high as 0.33 and R-squared was 0.09

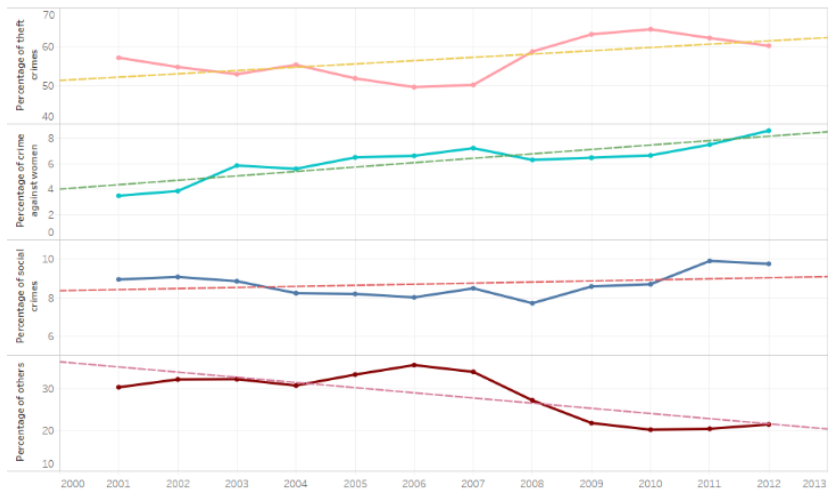

Fig 4:-Crime wise distribution in Delhi

Next is a dashboard in which filters are interactive i.e. the police can select the years which they want to study, then the number of IPC violations will be updated on the regions in Delhi and distribution of crime. Now, the police can find which region had most crimes. Further, if we want to study the percentage of each crime in a particular region or regions, we can click on the required regions and the crime distribution bar graph will update automatically. For example, when the crime was on the rise from 2008 to 2012, we found that South Delhi and South-East Delhi registered over 30,000 crimes. To find out what crimes happened there, selecting only these two regions produced an astonishing figure of $63.18 \%$ theft crimes. So, proper action needs to be taken to bring that figure down. Similar analysis can be performed to extract more information.

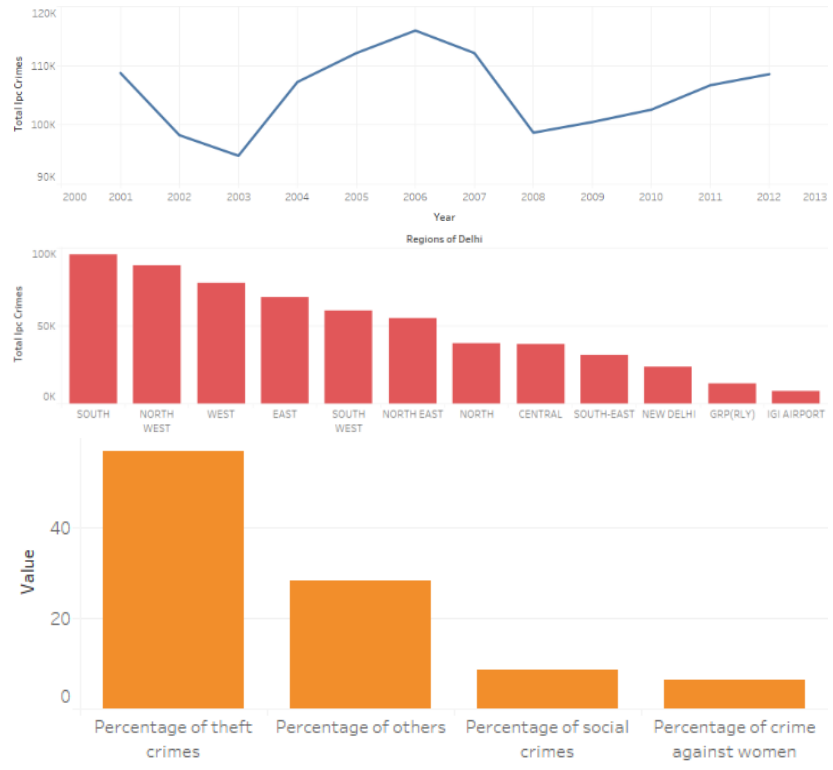

Fig 5:-Drill down crime region wise and crime wise 


\section{Conclusion and Future scope:-}

Contrasting difference between the two models should motive Indian government to make interactive dashboards. Intergraph is one enterprise which provides open BI platform and then can help provide dashboards, scorecards, reports, ad-hoc queries etc. [8]

One example of general report categories is shown in Table 1. Here, we found 4 general report categories viz:

1. Employee Activity: This report will monitor all police personnel activities like when they logged in and logged off from their duty, all activities performed on duty along with using GPS to pin point their location.

2. Calls for Service: This is the part where the control room of the department will work on. They will have interactive dashboards where all 1-0-0 calls will be managed and instant statistics like number of personals in demand, area from where maximum calls are coming, average time taken per call, average response time by Police etc.

3. Incidents/Events: Although all these attributes are already listed in FIR or any report made, but they are not open in the digital form to the people and this is where the data granularity is taken to the highest level. Personal information of the criminal shall however be removed for privacy and security purposes.

4. Unit Activity: All unit activity shall be monitored from the control room like where the PCR vehicle is stationed, which areas it is covering, the number of incidents it is reporting and which of them being solved instantly.

Table 1:-Types of reports and their attributes

\begin{tabular}{|l|l|}
\hline Report Category & \multicolumn{1}{|c|}{ Report Attributes } \\
\hline & $\bullet$ Logged on Duty Position \\
Employee Activity & $\bullet$ Officer Activity \\
& $\bullet$ Employee Workload \\
\hline Calls for Service & $\bullet$ Call Statistics \\
& $\bullet$ Demand Analysis \\
\hline & $\bullet$ Case Number \\
& $\bullet$ Event Demand \\
& $\bullet$ Event Register \\
& $\bullet$ Event Detail \\
& $\bullet$ Event Handling Time \\
& $\bullet$ Event Response Time \\
\hline Unit Activities & $\bullet$ Events by Location \\
\hline
\end{tabular}

Concluding all the facts, now we say that US cities like Baltimore, already imported their data on BI platform open for analysis and discussion. Hence, any person with good analytical skills can showcase their facts and findings and report it to the Police department.

However, this was not the case for India. In India, the information just lies there on the website with no promotion of analysis. One of the sparking difference between the two was the data granularity. US cities gave the highest level of granularity i.e. Baltimore Police gave information of each and every arrest, 9-1-1 call etc. and can extract a high number of facts and findings. With multiple cities participating in the same model, the data can be easily rolled up and the granularity can be decreased to get facts as a whole.

But, the Open Government Data platform of India provides the data with low level of granularity, hence making it difficult to analyse. No feature discussed in part 3.2 (features of Public Safety Model) was present. Consequently, the results obtained were also of low granularity. One reason for that is maybe of the corruption among the departments where Police departments tamper with the FIRs, evidence etc. [13,14].

Therefore, we strongly recommend the Indian government to step up and take an initiative to make dashboards (like discussed in Table 1) and automatically generated reports on a BI platform to make a safer India. 


\section{References:-}

1. MAPS: How Mapping Helps Reduce Crime and Improve Public Safety", nij.gov, 2016. [Online]. Available: http://www.nij.gov/topics/technology/maps/pages/reduce-crime.aspx

2. R. Sibley, "The benefits of criminal justice data: Beyond policing", Sunlight Foundation, 2015. [Online]. Available: https://sunlightfoundation.com/blog/2015/05/01/the-benefits-of-criminal-justice-data-beyondpolicing/

3. "What is Business Intelligence | IGI Global", Igi-global.com, 2016. [Online]. Available: http://www.igiglobal.com/dictionary/business-intelligence/3043

4. RANJAN, JAYANTHI. Business Intelligence: Concepts, Components, Techniques And Benefits.2005

5. "BPD Arrests | OpenBaltimore / City of Baltimore's Open Data Catalog",OpenBaltimore, 2016. [Online]. Available: https://data.baltimorecity.gov/Public-Safety/BPD-Arrests/3i3v-ibrt. [Accessed: 10- Oct- 2016]

6. "Georgia Tech uses data science to promote social good", News.gatech.edu, 2016. [Online]. Available: http://www.news.gatech.edu/2014/06/30/georgia-tech-uses-data-science-promote-social-good

7. J. Ratcliffe, Integrated intelligence and crime analysis. Washington, DC: Police Foundation, 2007.

8. Bringing Business Intelligence To Public Safety. 2016. Web. Available: www.intergraph.com/assets/pdf/BI_white_000.pdf

9. Boba, R. 2005. Crime Analysis and Crime Mapping. Thousand Oaks, CA: Sage.

10. "Calls for Service / OpenBaltimore / City of Baltimore's Open Data Catalog", Open Baltimore, 2016. [Online]. Available: https://data.baltimorecity.gov/Public-Safety/Calls-for-Service/xviu-ezkt. [Accessed: 10- Oct- 2016]

11. T. Newcombe, "How to Unlock Economic Benefits from Open Data: Part II", Govtech.com, 2016. [Online]. Available: http://www.govtech.com/data/How-to-Unlock-Economic-Benefits-from-Open-Data-Part-II.html

12. R. Luthra and M. Chhabra, "Criminal Analysis of Delhi 2001-2012 -Raghav | Tableau Public", Public.tableau.com, 2016. [Online]. Available: https://public.tableau.com/profile/raghav1588\#!/vizhome/CriminalAnalysis/CriminalAnalysisofDelhi2001-2012

13. D. Mohapatra, "Police accused of tampering FIR - Times of India", The Times of India, 2013. [Online]. Available: $\quad$ http://timesofindia.indiatimes.com/city/bhubaneswar/Police-accused-of-tamperingFIR/articleshow/25725970.cms

14. S. Narayan, "Court pulls up police for fabricating evidence", The Indian Express, 2014. [Online]. Available: http://indianexpress.com/article/cities/delhi/court-pulls-up-police-for-fabricating-evidence/

15. "District-Wise Crimes Under Various Sections of Indian Penal Code (IPC) Crimes | Open Government Data (OGD) Platform India". data.gov.in. N.p., 2016. [Online]. Available: https://data.gov.in/catalog/district-wisecrimes-under-various-sections-indian-penal-code-ipc-crime 